\title{
THE ROLE OF GRAVITATIONAL RADIATION IN THE EVOLUTION OF DWARF NOVAE
}

\author{
JOHN FAULKNER \\ Lick Observatory, U.S.A.
}

\begin{abstract}
Mechanisms promoting mass transfer include (i) envelope instability or (ii) nuclear evolution of the red star and (iii) gravitational radiation of orbital angular momentum. Growing observational evidence against (i) is supported by recent theoretical work on the medium and long-term response of stellar radii to mass-loss (Eggleton, Faulkner and Webbink, in progress). Since (ii) is in most cases too slow a process, (iii) is left as the best surviving explanation.
\end{abstract}

* See proceedings of IAU Symposium No. 66 . 